\title{
Integral models of unitary representations of current groups with values in semidirect products
}

\author{
A. M. Vershik 巴, M. I. Graev [2
}

\begin{abstract}
We describe a general construction of irreducible unitary representations of the group of currents with values in the semidirect product of a locally compact subgroup $P_{0}$ and a one-parameter group $\mathbb{R}_{+}^{*}=\{r: r>0\}$ of automorphisms of $P_{0}$. This construction is determined by a a faithful unitary representation of $P_{0}$ (canonical representation) whose images under the action of the group of automorphisms tend to the identity representation as $r \rightarrow 0$. We apply this construction to the groups of currents of the maximal parabolic subgroups of the groups of motions of the $n$-dimensional real and complex Lobachevsky spaces. The obtained representations of the groups of parabolic currents can be uniquely extended to the groups of currents with values in the semisimple groups $O(n, 1)$ and $U(n, 1)$. This gives a new description of the representations of the groups of currents of these groups constructed in the 70s and realized in the Fock space. The key role in our construction is played by the so-called special representation of the parabolic subgroup $P$ and the remarkable $\sigma$-finite measure (Lebesgue measure) $\mathcal{L}$ in the space of distributions.
\end{abstract}

To dear Israel Moiseevich Gelfand on the occasion of his 95th birthday

In 1972, on the initiative of I. M. Gelfand, a series of papers by three authors (I.M., M.I., A.M) on unitary representations of functional groups, or current groups, was started. The problem that was posed by I.M. to the first author of this paper in spring 1972 and that initiated this series of papers was to find out whether there exists a "multiplicative integral of representations" (see below) for the group $S L(2, R)$. Soon it became clear that the answer to this question is positive, and the first paper of the series [2] contained a description of several models of this representation. The main idea was to study a neighborhood of the identity representation of the group $S L(2, R)$ itself and the so-called special (infinitesimal) representation of this group, whose first cohomology is nontrivial. A multiplicative integral of representations is, in the authors' words, a "tensor product of infinitely many infinitesimal representations." In the subsequent joint papers, various generalizations of this construction (to simple Lie groups of rank 1, for groups of diffeomorphisms, etc.) were found, and techniques for working with such representations were developed. Starting from 2004 , the authors of this paper initiated a systematic study of integral models of representations of current groups based on a new interpretation of continual tensor product that is different from the Fock one and essentially exploits a remarkable $\sigma$-finite measure in the space of distributions. A systematic presentation of the whole area will be given in the book The Representation Theory of Current Groups by the three authors, which is now in preparation.

${ }^{1)}$ Supported by the grants NSh-2460.2008.1 and RFBR 08-01-00379.

${ }^{2)}$ Supported by the RFBR grant 07-01-00101a.

Date: August 9, 2008.

Key words and phrases. Current group, integral model, Fock representation, special representation, infinite-dimensional Lebesgue measure. 


\section{$\S 1$. Introduction}

We give a brief description of the theory of integral models of representations of current groups introduced in the previous paper by the same authors [10]. The integral models for the groups of currents of the parabolic subgroups of semisimple groups of rank 1 are used for constructing representations of the groups of currents of these semisimple groups themselves. We also establish a relation between the integral models and the corresponding representations of the groups of currents of semisimple groups of rank 1 and the Fock models considered earlier ([5, [6]).

By a group of currents we mean a group of measurable functions, on some space or manifold equipped with a measure, with values in some group of coefficients, endowed with the pointwise multiplication. Only those irreducible representations of current groups are of interest that are invariant under some group of automorphisms of the base space, for example, the group of rotations of the circle (in the case of loops) or the group of all measure-preserving transformations. In [2], such a representation (if it does exist) was called a multiplicative integral of representations, since in a sense it is a continuous tensor product of representations of the group of coefficients. The problem of existence of such a representation for a given group of coefficients and a given base space is far from being trivial. The general scheme of studying representations of current groups in a Fock space (i.e., in a Hilbert space endowed with a Fock factorization respected by representations) is presented in [1]. In particular, in this case the base of the current group can be an arbitrary measure space, and the group of coefficients $G$ must have an irreducible unitary representation with nontrivial first cohomology. As shown [13], the latter condition can be satisfied only if the identity representation of $G$ is not isolated in the set of irreducible unitary representations with the Fell topology (see [18]). Using the adopted terminology, one can say that $G$ must not satisfy Kazhdan's property $(\mathrm{T})$. An irreducible unitary representation $\pi$ with nontrivial first cohomology $H^{1}(G ; \pi)$ is called special. Among the semisimple Lie groups, only some groups of rank 1 have a special representation, namely, the groups of motions of the real and complex Lobachevsky spaces: $U(n, 1)$ and $O(n, 1)$. For the groups of currents with coefficients in these groups, the corresponding irreducible representations were first constructed in [2, 3]. They are realized in a Fock space, which is the exponential of a one-particle space, the latter being in turn a direct integral of special representations. The construction of any representation of a current group in a Fock space substantially uses a nontrivial 1-cocycle of the group of coefficients with values in a special representation of this group, whose existence guarantees the irreducibility of the representation of the current group. The Fock model is convenient, and it is used in most papers on the representation theory of infinite-dimensional groups. In this model, the operators of the maximal compact subgroup of currents are diagonalized. Note also that the structure of a Fock Hilbert space can be equivalently described with the help of the infinite-dimensional Gaussian measure ("white noise"), and that the model suggested below uses another remarkable measure in an infinite-dimensional space.

The paper [5] initiated the study of another model of representations of current groups, in which the operators of unipotent subgroups are diagonalized. This approach has gradually led to a quite new understanding of the nature of multiplicative integrals of representations and continuous tensor products. It is based on two principal observations. 
1) As in the case of the classical semisimple Lie groups, e.g., $S L(2, \mathbb{R})$, one should start constructing a representation of a group of semisimple currents with constructing a representation of currents of the maximal parabolic subgroup, and then extend it to the whole group of currents of the semisimple group.

2) In turn, the existence of a representation of the group of currents of the maximal parabolic subgroup of a semisimple group of rank 1 is due to the fact that there exists a remarkable $\sigma$-finite measure on the cone of discrete finite positive measures on a manifold that is invariant under the continuous analog of the Cartan subgroup, namely, the group of multiplicators with finite integral of the logarithm. This measure, which was introduced in [5], studied in [15, 14], and investigated in detail in the recent papers [11, 12], was called the infinite-dimensional Lebesgue measure. It allows us to drastically simplify all constructions of representations of current groups of semidirect products. Moreover, it proves to be closely related to many combinatorial, analytical, and probabilistic problems.

For the reason that will be clear from the construction, we call this type of models of representations of current groups integral models. In this paper, we present a coherent treatment of this alternative construction of integral models of representations of current groups of semidirect products, and show how they can be extended to the groups of currents of the groups of motions of the Lobachevsky spaces.

In brief, our model of continuous tensor products of Hilbert spaces and representations of groups of currents (loops) in these continuous tensor products is as follows:

a) we consider the space of countable linear positive combinations of $\delta$-measures on the base space with a finite sum of coefficients, endowed with the infinite-dimensional Lebesgue measure defined below [12, 11];

b) with each trajectory we associate a countable tensor product of Hilbert spaces and representations in these spaces, taken along this trajectory;

c) we define the direct integral of the constructed countable tensor products with respect to the Lebesgue measure on the space of trajectories.

Our construction yields representations of groups of currents (and, in particular, groups of loops) in a Hilbert space endowed with an additional structure, denoted by INT; these representations are equivalent to the representations constructed earlier in [2, [3] and realized in the Fock space EXP. The (unique up to a scalar) isomorphism between the spaces EXP and INT is of interest in itself.' One more advantage of this method of constructing representations of current groups is that the irreducibility of the representation of the parabolic group of currents, as well as the analysis of the special representation, becomes almost obvious. The only problem is to extend representations to the whole semisimple group of currents. This turns out to be possible for $O(n, 1)$ and $U(n, 1)$, and impossible for $S p(n, 1)$.

In Section 2 we define the class of semidirect products that serve as the groups of coefficients of current groups in our construction of integral models of representations. We introduce the notion of a canonical representation, which is used in the definition of a special

1) Note that this isomorphism preserves the structure of a Fock factorization of tensor products of Hilbert spaces. At the same time, there exist non-Fock factorizations in Hilbert spaces (see [17]), and hence there is a possibility to construct representations of current groups with factorizations nonisomorphic to the Fock one. As far as we know, this possibility has not yet been studied. 
representation of a semidirect product. In Section 3 we describe the main construction. Sections 4-5 contain formulas for the spherical function of the integral model and for the isomorphism between the integral model and the Fock model. In Section 6 we give a detailed description of the integral model in the simplest case, that of the parabolic subgroup of the group $S L(2, \mathbb{R})$, and construct an extension of this integral model to the whole group. In Sections 7-8 we do the same for the general case of the parabolic subgroups of the groups $U(n, 1)$ and $O(n, 1)$. For $U(n, 1)$, we should distinguish the cases of orthogonal and unitary special representations: in the unitary case, there arise projective unitary representations of the group of currents of $U(n, 1)$, also considered in [6]. There is a relation between these projective representations and unitary (nonprojective) representations of the current group $\widetilde{G}^{X}$, where $\widetilde{G}$ is the universal covering group of $U(n, 1)$ constructed in $[4$.

Note also that there exists a well-developed theory of projective highest weight representations for the case of groups of smooth loops with semisimple coefficients (the Kac-Moody theory). The same applies also to other representations of smooth currents. The proofs and the detailed version of the theory of integral models is presented in another paper, presently under preparation.

\section{$\S 2$. The original construction: special representations of semidirect products of groups}

We begin with a general scheme which applies, in particular, to parabolic subgroups of semisimple groups of rank 1. Consider the semidirect product $P=S \curlywedge P_{0}$ of a locally compact group $P_{0}$ and a one-parameter multiplicative group $S \cong \mathbb{R}_{+}^{*}$ of automorphisms of $P_{0}$. Denote by $g^{r}$ the image of an element $g \in P_{0}$ under an automorphism $r \in S$. In what follows, unless otherwise stated, the term "representation" means an orthogonal or unitary representation. The group of automorphisms also acts on representations $T$ of the group $P_{0}$; namely, every representation $T$ of the subgroup $P_{0}$ in a Hilbert space $H$ gives rise to a family of $T$-associated representations $T_{r}, r \in \mathbb{R}_{+}^{*}$, obtained from $T$ by automorphisms from the group $\mathbb{R}^{*}: T_{r}(g) \equiv T\left(g^{r}\right)$.

A cyclic representation $T$ of the subgroup $P_{0}$ is called canonical with respect to the group of automorphisms $S$ if there exists a unit cyclic vector $h \in H$ such that $\left\|T_{r}(g) h-h\right\|<$ $c(g) r$ for sufficiently small $r$ and any $g \in P_{0}$. A vector $h$ satisfying this property is called almost invariant with respect to the representation $T$. Obviously, in the Fell topology (see, e.g., [18]) on the space of representations, the representations $\left\{T_{r}\right\}$ tend to the identity representation as $r \rightarrow 0$. In terms of the matrix elements of $T$ (states) corresponding to cyclic vectors $h$, our definition reads as follows:

$$
\lim _{r \rightarrow 0} \frac{1-\varphi_{h}\left(g^{r}\right)}{r}=-\left.\frac{d \varphi_{h}\left(g^{r}\right)}{d r}\right|_{r=0} \equiv \psi(g)<\infty
$$

for all $g \in G$, where $\varphi_{h}(g)=\operatorname{Re}\{\langle T(g) h, h\rangle\}$ is a normalized positive definite function on the group $G$ (a "state"). ? $^{-}$

${ }^{2)}$ The function $\psi(\cdot)$ defined above is an unbounded conditionally positive definite function and is equal to the squared norm of the nontrivial cocycle in the special representation (see below). As to the terminology, note that in 10] a canonical representation was called summable, and in 2] canonical states for the group 
With every canonical representation $T$ of the subgroup $P_{0}$ we can associate a representation $\widetilde{T}$ of the group $P=\mathbb{R}_{+}^{*} \curlywedge P_{0}$ in the direct integral of the Hilbert spaces $H_{r} \equiv H$ with respect to the multiplicative Haar measure $r^{-1} d r$ on $\mathbb{R}_{+}^{*}$,

$$
\mathcal{H}=\int_{0}^{\infty} H_{r} r^{-1} d r
$$

i.e., in the space of sections $f(r)$ of the fiber bundle over $\mathbb{R}_{+}^{*}$ with fiber $H_{r}$ over a point $r \in \mathbb{R}_{+}^{*}$. The action of the operators $\widetilde{T}\left(g_{0}\right), g_{0} \in P_{0}$, on $\mathcal{H}$ is fiberwise: $\left(\widetilde{T}\left(g_{0}\right) f\right)(r)=$ $T_{r}\left(g_{0}\right) f(r)$; the operators $\widetilde{T}\left(r_{0}\right), r_{0} \in \mathbb{R}_{+}^{*}$, permute the fibers: $\left(\widetilde{T}\left(r_{0}\right) f\right)(r)=f\left(r_{0} r\right)$.

Lemma. The representation $\widetilde{T}$ of the group $P$ is a special representation (see [B]).

Indeed, by construction, this representation has nontrivial 1-cocycles $b: P \rightarrow \mathcal{H}$ of the form

$$
b(g, r)=\left(\widetilde{T}(g) f_{0}\right)(r)-f_{0}(r), \quad f_{0}(r)=\varphi(r) h_{r},
$$

where $h_{r}=h$ is a vector in $H$ almost invariant with respect to $T$ and $\varphi(r)$ is an arbitrary smooth function on $[0, \infty)$ such that $\int_{\epsilon}^{\infty} \varphi^{2}(r) r^{-1} d r<\infty$ for $\epsilon>0$ and $\varphi(0) \neq 0$.

Note that the 1-cocycles corresponding to functions $\varphi$ and $\varphi_{1}$ are equivalent if and only if $\varphi(0)=\varphi_{1}(0)$.

Examples of groups of the form $P=\mathbb{R}_{+}^{*} \wedge P_{0}$ for which the subgroup $P_{0}$ has canonical representations are the maximal parabolic subgroups of the groups $O(n, 1), U(n, 1)$, and $S p(n, 1)$. In the case of $O(n, 1)$, there is only one, up to passing to conjugate representations, canonical representation. In the cases of $U(n, 1)$ and $S p(n, 1)$, the group $P_{0}$ has countably many irreducible canonical representations. Each of them gives rise to a special irreducible representation of the group $P$. Note that among all special representations of the group $P \subset U(n, 1)$, only two unitary representations and one real representation can be extended to a special representation of the whole group $U(n, 1)$. The group $S p(n, 1)$ has no special representations at all.

\section{$\S 3$. Construction of the integral model of representations (INT)}

The current group $P^{X}$, where $P$ is a locally compact group and $X$ is a space with a probability measure $m$, is the group of bounded measurable mappings $X \rightarrow P$ endowed with pointwise multiplication. In [10], for groups $P$ of the form $P=\mathbb{R}_{+}^{*} \wedge P_{0}$, for each canonical representation $T$ of the subgroup $P_{0}$ in a space $H$ and a vector $h \in H$ almost invariant with respect to $T$, we defined a representation of the current group $P^{X}$, which was called the integral model. By analogy with Fock models EXP (see [5]), we will denote this representation of the group $P^{X}$ by INT $T$, and the space in which it is realized, by INT $H$, or, in more detail, $\operatorname{INT}(H, h)$.

The construction of the representation INT $T$ of the current group $P^{X}$ for $P=\mathbb{R}_{+}^{*} \curlywedge P_{0}$ is similar to the construction of a special representation of the group $P$ from a canonical representation $T$ of the subgroup $P_{0}$. First of all, in this construction, the space $\mathbb{R}_{+}^{*}$ is

$P S L(2, R)$ were defined as states that are invariant with respect to a compact subgroup and satisfy a close condition. Here we somewhat extend the notion of a canonical state using the same term. 
replaced by the cone of real positive finite atomic measures on the space $(X, m)$ :

$$
l_{+}^{1}(X)=\left\{\xi=\sum_{k=1}^{\infty} r_{k} \delta_{x_{k}} \mid r_{k}>0, \sum_{k} r_{k}<\infty\right\} ;
$$

in what follows, elements of this space will be denoted by $\xi=\left\{r_{k}, x_{k}\right\}$. Instead of the multiplicative Haar measure $r^{-1} d r$ on $\mathbb{R}_{+}^{*}$, we consider the infinite-dimensional $\sigma$-finite measure $d \mathcal{L}(\xi)$ on $l_{+}^{1}(X)$. This measure (see [11]), which is an infinite-dimensional analog of the Lebesgue measure on the octant $\mathbb{R}_{+}^{n}$, is uniquely determined by its Laplace transform:

$$
\Psi_{\mathcal{L}}(f) \equiv \int_{l_{+}^{1}(X)} \exp \left(-\sum_{k} r_{k} f\left(x_{k}\right)\right) d \mathcal{L}(\xi)=\exp \left(-\int_{X} \log f(x) d m(x)\right),
$$

where $f$ is an arbitrary nonnegative measurable function on $(X, m)$ with $\int \log f(x) d m(x)<$ $\infty$. Note that, like the classical Laplace transform, equation (2) makes sense also for complex-valued functions $f$ with nonnegative real component.

The construction of the integral model essentially uses only two properties of the measure $\mathcal{L}$ : its projective invariance with respect to the group of multiplicators $M_{a}$ (namely, $d \mathcal{L}\left(M_{a} \xi\right)=\varphi(a) d \mathcal{L}(\xi)$, where $\left.\varphi(a)=e^{\frac{1}{2} \int_{X} \log a(x) d m(x)}\right)$ and its ergodicity with respect to this group. The invariance follows directly from the formula for the Laplace transform; for a proof of the ergodicity, see [12]. In particular, the projective invariance of $\mathcal{L}$ implies the orthogonality (respectively, unitarity) of the representation INT $T$, and the ergodicity of $\mathcal{L}$ implies the irreducibility of INT $T$. For a detailed study of the properties of this remarkable measure, see [11], [12].

Further, in the construction of the integral model, the spaces $H_{r}, r \in \mathbb{R}_{+}^{*}$ (which are the spaces of representations of the subgroup $P_{0}$ ) are replaced by the countable tensor products $H_{\xi}=\otimes_{k=1}^{\infty} H_{r_{k}}, \xi \in l_{+}^{1}(X)$, with stabilizing almost invariant vectors $h \in H$ (which are the spaces of representations $T_{\xi}$ of the group $\left.P_{0}^{X}\right)$.

By definition, the countable tensor product $H_{\xi}=\otimes_{k=1}^{\infty} H_{r_{k}}$ with stabilizing almost invariant vector $h \in H$ is the completion of the inductive limit of the spaces $\otimes_{k=1}^{n} H_{r_{k}}$ with respect to the embeddings $x \in \otimes_{k=1}^{n} H_{r_{k}} \mapsto x \otimes h_{r_{n+1}} \in \otimes_{k=1}^{n+1} H_{r_{k}}, h_{r_{n+1}}=h$. The action of the operators corresponding to the subgroup $P_{0}^{X}$ on $H_{\xi}$ is given by the formula

$$
T_{\xi}(g(\cdot))\left(\otimes_{k=1}^{\infty} f_{k}\right)=\otimes_{k=1}^{\infty}\left(T_{r_{k}}\left(g\left(x_{k}\right)\right) f_{k}\right) .
$$

Note that the representations $T_{\xi}$ are well defined only for canonical representations $T$ of the subgroup $P_{0}$.

The space INT $H$ of the integral model $U=$ INT $T$ is defined, by analogy with the space $\mathcal{H}=\int_{0}^{\infty} H_{r} r^{-1} d r$ of the representation of $P$ associated with a canonical representation $T$ of $P_{0}$, as the direct integral of the Hilbert spaces $H_{\xi}$ with respect to the measure $\mathcal{L}$ :

$$
\operatorname{INT} H=\int_{l_{+}^{1}(X)}^{\oplus} H_{\xi} d \mathcal{L}(\xi), \quad H_{\xi}=\otimes_{k=1}^{\infty} H_{r_{k}},
$$

i.e., as the space of sections $F(\xi)=F\left(\left\{r_{k}, x_{k}\right\}\right)$ of the fiber bundle over $l_{+}^{1}(X)$ with fiber $H_{\xi}$ over a point $\xi=\left\{r_{k}, x_{k}\right\}$. The action of the subgroup $P_{0}^{X}$ on the spaces $H_{\xi}$ induces a 
representation $U=\operatorname{INT} T$ of this group in the whole space INT $H$ :

$$
\operatorname{INT} T=\int_{l_{+}^{1}(X)}^{\oplus} T_{\xi} d \mathcal{L}(\xi), \quad \text { where } \quad T_{\xi}=\otimes_{k=1}^{\infty} T_{r_{k}, x_{k}} ;
$$

in more detail: $(U(g(\cdot)) F)(\xi)=\widetilde{T}_{\xi}\left(g(\cdot) F(\xi)\right.$. The extension of this representation of $P_{0}^{X}$ to the whole group $P^{X}$ is given by the formula

$$
\left(U\left(r_{0}(\cdot)\right) F\right)(\xi)=e^{\frac{1}{2} \int_{X} \log r_{0}(x) d m(x)} F\left(r_{0}(\cdot) \xi\right) \quad \text { for } \quad r_{0} \in\left(\mathbb{R}_{+}^{*}\right)^{X} .
$$

It follows from the definition that the representation INT $T$ of the group $P^{X}$ is invariant under transformations of the space $X$ preserving the measure $m$.

Theorem 1. ([10]) If a canonical representation $T$ of the subgroup $P_{0}$ is irreducible, then the corresponding representations $\widetilde{T}$ and $U=\operatorname{INT} T$ of the groups $P$ and $P^{X}$, respectively, are irreducible.

Remark. The construction of the integral model also makes sense if we replace the probability measure $m$ on $X$ with any positive finite measure (with $m(X)=\theta$ ) or, which is equivalent, introduce the coefficient $\theta>0$ into the exponent in the definition of the Laplace transform of the measure $\mathcal{L}$. The one-parameter family, thus defined, of representations of $P^{X}$ still satisfies Theorem 1). For simplicity, here we restrict ourselves with the case $\theta=1$.

Let us define the spherical function of the representation $U=\operatorname{INT} T$ of the group $P^{X}$ associated with a canonical representation $T$ of the subgroup $P_{0}$ in a space $H$ as the following function on $P^{X}$ :

$$
\Psi(g)=\langle U(g) \Omega, \Omega\rangle,
$$

where $\Omega$ is a unit vector in the space $\widetilde{H}=\operatorname{INT} H$ of the form

$$
\Omega(\xi)=\otimes_{k=1}^{\infty}\left(e^{-\frac{1}{2} r_{k}} h_{r_{k}}\right), \quad h_{r_{k}}=h \quad \text { for } \quad \xi=\left\{r_{k}, x_{k}\right\} \in D_{+}(X) .
$$

M:1 Theorem 2. The spherical function of the representation $U$ is given by the formula

$$
\Psi(g)=\exp \left(\int_{X}\left(i \operatorname{Im}\left\langle b(g(x)), f_{0}\right\rangle-\frac{1}{2} \| b\left(g(x) \|^{2}\right) d m(x)\right),\right.
$$

where $f_{0}(r)=e^{-\frac{r}{2}} h_{r}$ and $b(g)=\left(\widetilde{T}(g) f_{0}\right)(r)-f_{0}(r)$ is the 1-cocycle of the representation $\widetilde{T}$ of the group $P$ associated with $T$, see (四).

\section{$\S 4$. Relation between integral models and Fock representations}

Given an arbitrary locally compact group $G$, each pair $(\widetilde{T}, b)$, where $\widetilde{T}$ is a special representation of $G$ in a space $\mathcal{H}$ and $b: G \rightarrow \mathcal{H}$ is a nontrivial 1-cocycle, gives rise to a Fock model of the corresponding representation of the current group $G^{X}$, see [5]. It is realized in the Hilbert space $\operatorname{EXP} \mathcal{H}^{X}=\oplus_{k=0}^{\infty} S^{k} \mathcal{H}^{X}$, where $S^{k}$ stands for the $k$ th symmetric tensor power and $\mathcal{H}^{X}=\int_{X}^{\oplus} \mathcal{H}_{x} d m(x), \mathcal{H}_{x}=\mathcal{H}$. The operators of this representation are defined on the total subset of vectors of the form $\operatorname{EXP} v=\mathbb{I} \oplus v \oplus \frac{1}{\sqrt{2 !}} v \otimes v \oplus \frac{1}{\sqrt{3 !}} v \otimes v \otimes v \oplus \cdots$ by the formula

$$
U(g) \operatorname{EXP} v=\exp \left(-\frac{1}{2}\left\|b^{X}(g)\right\|^{2}-\left(\widetilde{T}^{X}(g) v, b^{X}(g)\right)\right) \operatorname{EXP}\left(\widetilde{T}^{X}(g) v+b^{X}(g)\right) .
$$


Here $\widetilde{T}^{X}$ and $b^{X}(g)$ are the representation of the group $G^{X}$ in the space $\mathcal{H}^{X}$ and the 1cocycle $b^{X}: G^{X} \rightarrow \mathcal{H}^{X}$ generated, respectively, by the representation $\widetilde{T}$ of the group $G$ and the 1-cocycle $b: G \rightarrow \mathcal{H}$. The operators $U\left(g_{1} g_{2}\right)$ and $U\left(g_{1}\right) U\left(g_{2}\right)$ in the Fock representation are related by the formula $U\left(g_{1} g_{2}\right)=\exp \left(i \operatorname{Im} \int_{X} a\left(g_{1}(x), g_{2}(x)\right) d m(x)\right) U\left(g_{1}\right) U\left(g_{2}\right)$, where $a\left(g_{1}, g_{2}\right)=\left(\widetilde{T}\left(g_{1}\right) b\left(g_{2}\right), b\left(g_{1}\right)\right)$. Thus, in the case of unitary representations $\widetilde{T}$, the Fock representations are, in general, projective. However, for groups of the form $P=\mathbb{R}_{+}^{*}<P_{0}$, the 2-cocycle $a\left(g_{1}, g_{2}\right)$ is trivial, so that these representations are projective equivalent to ordinary (nonprojective) representations.

M:2 Theorem 3. Let $T$ be a canonical representation of the group $P_{0}$ and $\widetilde{T}$ be the special representation of the group $P$ associated with $T$ (see above). Then the integral model INT $T$ of the corresponding representation of the group $P^{X}$ is projective equivalent to the Fock representation of this group associated with the same representation $\widetilde{T}$ and the 1-cocycle of the form (四) with $f_{0}(r)=e^{-\frac{r}{2}} h_{r}$.

\section{$\S 5$. Integral models of representations of the subgroup $P \subset S L(2, \mathbb{R})$ of triangular matrices and their extensions to the group $S L(2, \mathbb{R})^{X}$}

Let us write elements of the subgroup $P \subset S L(2, \mathbb{R})$ of triangular matrices $g=$ $\left(\begin{array}{cc}\epsilon^{-1} & 0 \\ \gamma & \epsilon\end{array}\right)$ as pairs $(\epsilon, \gamma)$ with the multiplication law $\left(\epsilon_{1}, \gamma_{1}\right)\left(\epsilon_{2}, \gamma_{2}\right)=\left(\epsilon_{1} \epsilon_{2}, \gamma_{1} \epsilon_{2}^{-1}+\epsilon_{1} \gamma_{2}\right)$. The group $P$ can be presented as the semidirect product $P=\mathbb{R}_{+}^{*} \wedge P_{0}$, where $P_{0}$ is the commutative group consisting of the elements $( \pm 1, \gamma)$.

Up to passing to conjugate representations, the group $P_{0}$ has two unitary irreducible canonical representations $T^{ \pm}$in one-dimensional spaces $H^{ \pm}$and one orthogonal representation $T^{0}$ in a two-dimensional space $H^{0}$. The representations $T_{r}^{ \pm}$in the spaces $H_{r}^{ \pm}=H^{ \pm}$ are defined by the formulas $T_{r}^{ \pm}(1, \gamma)=e^{ \pm i r^{2} \gamma} \mathrm{id}, T_{r}^{ \pm}( \pm 1,0)=\mathrm{id}$. Accordingly, the countable tensor products $H_{\xi}^{ \pm}, \xi=\left\{r_{k}, x_{k}\right\}$, in which the current group $P_{0}^{X}$ acts, are also one-dimensional, so that the spaces INT $H^{ \pm}$of the representations $U^{ \pm}=$INT $T^{ \pm}$of the group $P^{X}$ are the Hilbert spaces of complex-valued functionals $F^{ \pm}(\xi)=F\left(\left\{r_{k}, x_{k}\right\}\right)$ on $l_{+}^{1}(X)$ with the norm

$$
\|F\|^{2}=\int_{l_{+}^{1}(X)}|F(\xi)|^{2} d \mathcal{L}(\xi) .
$$

The operators of these representations are identical on the center of $P^{X}$ and are uniquely determined by the formulas

$$
\begin{gathered}
\left(U^{ \pm}(1, \gamma(\cdot)) F^{ \pm}\right)(\xi)=e^{ \pm i \sum r_{k}^{2} \gamma\left(x_{k}\right)} F^{ \pm}(\xi), \\
\left(U^{ \pm}\left(r_{0}(\cdot), 0\right) F\right)(\xi)=e^{\frac{1}{2} \int_{X} \log r_{0}(x) d m(x)} F^{ \pm}\left(r_{0}(\cdot) \xi\right) .
\end{gathered}
$$

The orthogonal representations $T_{r}^{0}$ are realized in the two-dimensional real subspaces $H_{r}^{0} \subset H_{r}^{+} \oplus H_{r}^{-}$of vectors of the form $(f, \bar{f})$; the corresponding operators are the restrictions of the operators $T_{r}^{+}(g) \oplus T_{r}^{-}(g)$ to these subspaces. Hence the spaces $H_{\xi}^{0}$ of the representations of $P_{0}^{X}$ are countable tensor products of two-dimensional real spaces, and the integral model $U^{0}=\operatorname{INT} T^{0}$ of the corresponding representation of $P^{X}$ acts in the direct integral of these spaces with respect to the measure $\mathcal{L}$ : INT $H^{0}=\int_{D_{+}(X)} H_{\xi}^{0} d \mathcal{L}(\xi)$. 
The operators $U^{0}\left(r_{0}(\cdot), 0\right)$ of this representation are given by the same formula (6)), and the operators $U^{0}(1, \gamma(\cdot))$ are given by the formula

$$
U^{0}(1, \gamma(\cdot))\left(\otimes\left(f_{k}, \bar{f}_{k}\right)\right)=\otimes_{k=1}^{\infty}\left(e^{i r_{k}^{2} \gamma\left(x_{k}\right)} f_{k}, e^{-i r_{k}^{2} \gamma\left(x_{k}\right)} \bar{f}_{k}\right) .
$$

Note that under the natural embedding INT $H^{0} \subset \operatorname{INT}\left(H^{+} \oplus H^{-}\right)$, the operators $U^{0}(g)$ are the restrictions to INT $H^{0}$ of the operators $U(g)$ in the space $\operatorname{INT}\left(H^{+} \oplus H^{-}\right)$.

Let us describe the extensions of the representations $U^{ \pm}$and $U^{0}$ of the current group $P^{X}$ to the group $S L(2, \mathbb{R})^{X}$.

Consider a homogeneous space of the group $G=S L(2, \mathbb{R})$, namely, the upper complex half-plane $L$ endowed with the following action of $G: z \rightarrow g z=\frac{\delta z+\gamma}{\beta z+\alpha}$ for $g=\left(\begin{array}{ll}\alpha & \beta \\ \gamma & \delta\end{array}\right)$ (the Lobachevsky plane). Denote by $L^{X}$ the space of bounded functions $z: X \rightarrow L$. Note that the integral $\int_{X} \log |z(x)| d m(x)$ converges for every function $z \in L^{X}$, since $z$ is bounded. The action of the group $G$ on $L$ induces the pointwise action of the group $G^{X}$ on $L^{X}$.

Further, consider on $L \times L$ the function

$$
c\left(z_{1}, z_{2}\right)=\log \left(-i\left(z_{1}-\overline{z_{2}}\right)\right)=\log \left[\left(v_{1}+v_{2}\right)-i\left(u_{1}-u_{2}\right)\right], \quad z_{k}=u_{k}+i v_{k},
$$

and define functions $u^{ \pm}(g, z)$ on $G \times L$ by the formulas

$$
u^{+}(g, z)=\overline{u^{-}(g, z)}=c\left(g z, g z_{0}\right)-c\left(z, z_{0}\right)-\frac{1}{2}\left(c\left(g z_{0}, g z_{0}\right)-c\left(z_{0}, z_{0}\right)\right),
$$

where we have denoted $z_{0}=i$.

In order to extend the representations $U^{ \pm}=\operatorname{INT} T^{ \pm}$of the group $P^{X}$ to the group $S L(2, \mathbb{R})^{X}$, with each function $z \in L^{X}$ we associate the following functionals on $l_{+}^{1}(X)$ :

$$
F_{z}^{+}(\xi)=e^{i \sum r_{k}^{2} z\left(x_{k}\right)}, \quad F_{z}^{-}(\xi)=\overline{F_{z}^{+}(\xi)}=e^{-i \sum r_{k}^{2} \overline{z\left(x_{k}\right)}} .
$$

These functionals lie in the spaces INT $H^{ \pm}$and form total subsets in these spaces.

We define the action of the operators $U^{ \pm}(g)$ of the current group $S L(2, \mathbb{R})^{X}$ on the sets of functionals of the form $F_{z}^{ \pm}$by the following formula:

$$
U^{ \pm}(g) F_{z}^{ \pm}=e^{\frac{1}{2} \int_{X} u^{ \pm}(g(x), z(x)) d m(x)} F_{g z}^{ \pm},
$$

where $u^{ \pm}(g, z)$ is given by (9).

Theorem 4. The operators $U^{ \pm}(g)$ defined by (10) can be extended to unitary operators on the corresponding spaces INT $H^{ \pm}$and determine projective representations of the group $G^{X}$ in INT $H^{ \pm}$. The restrictions of these representations to the subgroup $P^{X}$ coincide with the original representations of this subgroup.

In order to extend the orthogonal representation $U^{0}=\operatorname{INT} T^{0}$ of the group $P^{X}$ in the space INT $H^{0}$ to the group $S L(2, \mathbb{R})^{X}$, with each function $z \in L^{X}$ we associate the following functional on $l_{+}^{1}(X)$ :

$$
F_{z}^{0}(\xi)=\otimes_{k=1}^{\infty}\left(2^{-1 / 2} e^{i r_{k}^{2} z\left(x_{k}\right)}, 2^{-1 / 2} e^{-i r_{k}^{2} \overline{z\left(x_{k}\right)}}\right) .
$$

The functionals $F_{z}^{0}$ lie in the space INT $H^{0}$ and form a total set in this space. 
We define the action of the operators $U^{0}(g)$ of the group $P^{X}$ on the set of functionals of the form $F_{z}^{0}$ by the formula

$$
U^{0}(g) F_{z}^{0}=e^{\frac{1}{2} \operatorname{Re} \int_{X} u^{ \pm}(g(x), z(x)) d m(x)} F_{g z}^{0},
$$

where $u^{ \pm}(g, z)$ is still given by (9) .

Theorem 5. The operators $U^{0}(g)$ can be extended to orthogonal operators on INT $H^{0}$ and determine an orthogonal representation of the group $G^{X}$ in INT $H^{0}$. Its restriction to the subgroup $P^{X}$ coincides with the original representation of $P^{X}$. The obtained representation of the group $S L(2, \mathbb{R})^{X}$ is equivalent to the representation constructed in [2].

\section{$\S 6$. Extension of the integral models of representations of the maximal parabolic subgroups of $O(n, 1), U(n, 1)$ to the whole groups: the orthogonal version}

It is known (see, e.g., [8]) that each of the groups $O(n, 1)$ and $U(n, 1)$ has a unique orthogonal irreducible special representation. Its restriction to the maximal parabolic subgroup $P$ is also irreducible and associated with a canonical orthogonal representation of the subgroup $P_{0}$ appearing in the decomposition $P=\mathbb{R}_{+}^{*} \wedge P_{0}$; denote this canonical orthogonal representation by $T^{0}$. The question arises whether it is possible to extend the orthogonal representation INT $T^{0}$ of the group $P^{X}$ to a representation of the group $O(n, 1)^{X}$ or $U(n, 1)^{X}$, respectively.

Theorem 6. The integral models INT $T^{0}$ of orthogonal representations of the groups $P^{X}$, where $P \subset O(n, 1)$ or $P \subset U(n, 1)$, can be extended up to orthogonal representations of the groups $O(n, 1)^{X}$ and $U(n, 1)^{X}$ that are equivalent to their Fock representations described in [5]. The corresponding intertwining operator is generated by the mapping $\Omega \mapsto \operatorname{EXP} 0$ of the cyclic vectors.

The assertion of the theorem follows from the coincidence of the spherical functions of the corresponding representations of the group $P^{X}$.

In the case of the group $U(n, 1)$, its special orthogonal irreducible representation can be endowed with the structure of a unitary representation in two ways. The restrictions of the special unitary representations of $U(n, 1)$ thus obtained to the subgroup $P$ are irreducible and associated with canonical unitary representations $T^{+}$and $T^{-}$of the subgroup $P_{0}$.

Theorem 7. The integral models INT $T^{ \pm}$of unitary representations of the group $P^{X}$ associated with the canonical unitary representations $T^{ \pm}$of the subgroup $P_{0}$ can be extended up to projective unitary representations of the current group $U(n, 1)^{X}$ that are projective equivalent to its Fock projective unitary representations described in [6].

The extensions thus obtained are new models of representations of the current groups $O(n, 1)^{X}$ and $U(n, 1)^{X}$, in which the representation space is realized as the direct integral of $P_{0}^{X}$-invariant subspaces with respect to the measure $\mathcal{L}$, and elements from $\left(\mathbb{R}_{+}^{*}\right)^{X} \subset P^{X}$ permute these subspaces. 


\section{$\S 7$. Extension of the integral models of representations of the maximal parabolic subgroup of $U(n, 1)$ to the whole group: the unitary version}

Let us realize $U(n, 1)$ as the group of linear transformations in $\mathbb{C}^{n+1}$ preserving the Hermitian form $x_{1} \bar{x}_{n+1}+x_{n+1} \bar{x}_{1}+\left|x_{2}\right|^{2}+\ldots+\left|x_{n}\right|^{2}$, and let us represent elements $g \in$ $U(n, 1)$ in a block form: $g=\left\|g_{i j}\right\|_{i, j=1,2,3}$, where the matrices on the diagonal are of orders $1, n-1$, and 1 , respectively. In this realization, $P$ is the subgroup in $U(n, 1)$ consisting of all lower triangular block matrices. It can be presented as the semidirect product $P=\mathbb{R}_{+}^{*} \wedge P_{0}$, where $P_{0}=D_{0} \curlywedge N, N$ is the Heisenberg group of order $2 n-1$ realized as the group of pairs $(t, z) \in \mathbb{R} \otimes \mathbb{C}^{n-1}$, and $D_{0} \cong U(1) \times U(n-1)$.

In the construction of the integral models INT $T^{ \pm}$and INT $T^{0}$, the canonical unitary representations $T^{ \pm}$of the group $P_{0}$ are realized in the spaces $H^{ \pm}$, respectively, of entire analytical and entire anti-analytical functions $f(z)$ on $\mathbb{C}^{n-1}$ with the norm $\|f\|^{2}=\int_{\mathbb{C}^{n-1}}|f(z)|^{2} e^{-z z^{*}} d \mu(z)$ (for the action of $P_{0}$ on $H^{ \pm}$, see $[\mathbb{8}]$ ). The orthogonal canonical representation $T^{0}$ is realized in the subspace $H^{0} \subset H^{+} \oplus H^{-}$of vectors of the form $(f, \bar{f})$.

According to the general definition, the representations $U^{ \pm}=\mathrm{UNT} T^{ \pm}$and $U^{0}=$ UNT $T^{0}$ of the group $P^{X}$ are realized in the space INT $H^{ \pm}=\int_{D_{+}(X)}^{\oplus} H_{\xi}^{ \pm} d \mathcal{L}(\xi)$, where $H_{\xi}^{ \pm}=\otimes_{k=1}^{\infty} H_{r_{k}}^{ \pm}$, and in the space INT $H^{0}=\int_{D_{+}(X)}^{\oplus} H_{\xi}^{0} d \mathcal{L}(\xi)$, where $H_{\xi}^{0}=\otimes_{k=1}^{\infty} H_{r_{k}}^{ \pm}$, respectively.

The extensions of these representations to the whole group $U(n, 1)^{X}$ can be constructed in a way similar to the case of $S L(2, \mathbb{R})$. Namely, instead of the Lobachevsky plane, we consider the homogeneous space $L$ of the group $U(n, 1)$ that is equivalent to the disk in $\mathbb{C}^{n}$ (the $n$-dimensional complex Lobachevsky space):

$$
L=\left\{v=(a, b) \in \mathbb{C} \oplus \mathbb{C}^{n-1} \mid a+\bar{a}+b^{*} b<0\right\}, \quad b^{*} b=\sum \bar{b}_{i} b_{i}
$$

( $b$ is a column vector). The action of the group $U(n, 1)$ on $L$ is given by the formula $g(a, b)=\left(a^{\prime}, b^{\prime}\right)$, where

$$
\begin{aligned}
& a^{\prime}=\left(g_{11}+g_{12} b+g_{13} a\right)^{-1}\left(g_{31}+g_{32} b+g_{33} a\right), \\
& b^{\prime}=\left(g_{11}+g_{12} b+g_{13} a\right)^{-1}\left(g_{21}+g_{22} b+g_{23} a\right) .
\end{aligned}
$$

Consider the following function on $L \times L$ :

$$
c\left(v_{1}, v_{2}\right)=\log \left(-a_{1}-\bar{a}_{2}-b_{2}^{*} b_{1}\right) \quad \text { for } \quad v_{i}=\left(a_{i}, b_{i}\right) \in L .
$$

Further, denote by $L^{X}$ the set of measurable bounded mappings $v: X \rightarrow L$ with the pointwise action $v \mapsto g v$ of the group $P^{X}$. Note that the integral $\int_{X} c(v(x), v(x)) d m(x)$ converges for every $v \in L^{X}$ since $v \in L^{X}$ is bounded.

With each triple $v=(a, b) \in L^{X}, r \in \mathbb{R}_{+}^{*}$, and $x \in X$, we associate a vector $f_{v, r, x} \in H_{r}$ :

$$
f_{v, r, x}(z)=\exp \left(r^{2} a(x)+r(z, b(x))\right), \quad(z, b)=\sum z_{i} b_{i}
$$

with elements $v \in L^{X}$ we associate the following functionals $F_{v}^{ \pm}(\xi)$ and $F_{v}^{0}(\xi)$ on $l_{+}^{1}(X)$ :

$$
F_{v}^{+}(\xi)=\overline{F_{v}^{-}(\xi)}=\otimes_{k=1}^{\infty} f_{v, r_{k}, x_{k}}, \quad F_{v}^{0}(\xi)=\otimes_{k=1}^{\infty}\left(2^{-1 / 2} f_{v, r_{k}, x_{k}}, 2^{-1 / 2} \overline{f_{v, r_{k}, x_{k}}}\right) .
$$


These functionals lie in the spaces INT $H^{ \pm}$and INT $H^{0}$, respectively, and form total subsets in these spaces.

On these sets we define the actions of the operators $U^{ \pm}(g)$ and $U^{0}(g)$ by the same formulas (10) and ([1), as in the case of the group $S L(2, \mathbb{R})$, in which $z$ should be replaced by $v$, the function $c\left(z_{1}, z_{2}\right)$ should be replaced by the function $c\left(v_{1}, v_{2}\right)$ introduced above, and instead of $z_{0}=i$ one should write $v_{0}=(-1,0)$.

Theorem 8. The operators $U^{ \pm}(g)$ and $U^{0}(g)$ can be extended to unitary and orthogonal operators in the spaces INT $H^{ \pm}$and INT $H^{0}$, respectively; they determine extensions of the original representations of the group $P^{X}$ up to projective unitary and orthogonal representations of the group $U(n, 1)^{X}$, respectively.

Translated by N. V. Tsilevich.

\section{References}

[1] H. Araki. Factorisable representations of the current algebra. Publ. RIMS Kyoto Univ., Ser. A5, No. 3 (1970), 361-422.

[2] A. M. Vershik, I. M. Gelfand, and M. I. Graev. Representations of the group $S L(2, R)$, where $R$ is a ring of functions. Uspekhi Mat. Nauk 28, No. 5 (1973), 83-128. English translation in: Representation Theory, London Math. Soc. Lect. Note Ser. 69, Cambridge Univ. Press, 1982, pp. 15-60.

[3] A. M. Vershik, I. M. Gelfand, and M. I. Graev. Irreducible representations of the group $G^{X}$ and cohomology. Funct. Anal. Appl. 8 (1974), 151-153.

[4] F. A. Berezin. Representations of the continuous direct product of universal coverings of the group of motions of a complex ball. Trans. Mosc. Math. Soc. 36 (1979), 281-298.

[5] I. M. Gelfand, M. I. Graev, and A. M. Vershik. Models of representations of current groups. In: Representations of Lie Groups and Lie Algebras (A. A. Kirillov, ed.), Akadémiai Kiadó, Budapest, 1985, pp. 121-179.

[6] I. M. Gelfand and M. I. Graev. Special representations of the group $\mathrm{SU}(n, 1)$ and projective unitary representations of the current group SU $(n, 1)^{X}$. Russian Acad. Sci. Dokl. Math. 48, No. 2 (1994), 291-295.

[7] A. M. Vershik and M. I. Graev. A commutative model of a representation of the group $\mathrm{O}(n, 1)^{X}$ and a generalized Lebesgue measure in a distribution space. Funct. Anal. Appl. 39, No. 2 (2005), 81-90.

[8] A. M. Vershik and M. I. Graev. Structure of the complementary series and special representations of the groups $O(n, 1)$ and $U(n, 1)$. Russian Math. Surveys 61, No. 5 (2006), 799-884.

[9] M. I. Graev and A. M. Vershik. The basic representation of the current group $O(n, 1)^{X}$ in the $L^{2}$ space over the generalized Lebesgue measure. Indag. Math. 16, No. 3/4 (2005), 499-529.

[10] A. M. Vershik and M. I. Graev. Integral models of representations of current groups. Funkts. Anal. $i$ Prilozh. 42, No. 1 (2008), 22-32.

[11] A. M. Vershik. Does there exist the Lebesgue measure in the infinite-dimensional space? Proc. Steklov Inst. Math. 259 (2007), 256-281.

[12] A. Vershik. Invariant measures for continual Cartan subgroup. J. Funct. Anal. (2008).

[13] A. M. Vershik and S. I. Karpushev. Cohomology of groups in unitary representations, the neighborhood of the identity, and conditionally positive definite functions. Math. in USSR 47 (1984), 513-526.

[14] A. M. Vershik and N. V. Tsilevich. Fock factorizations and decompositions of the $L^{2}$ spaces over general Levy processes. Russian Math. Surveys 58, No. 3 (2003), 427-472.

[15] N. Tsilevich, A. Vershik, and M. Yor. An infinite-dimensional analogue of the Lebesgue measure and distinguished properties of the gamma process. J. Funct. Anal. 185, No. 1 (2001), 274-296.

[16] R. S. Ismagilov. Representations of Infinite-Dimensional Groups. Transl. Math. Monographs 152. Amer. Math. Soc., 1996.

Moscow, 1974. 
TsV [17] B. Tsirelson and A. Vershik. Examples of nonlinear continuous tensor product of measure spaces and non-Fock factorizations. Rev. Math. Phys. 10, No. 1 (1998), 81-145.

XR [18] E. Hewitt and K. A. Ross. Abstract Harmonic Analysis, Vol. II. Springer-Verlag, New York-Berlin, 1970.

A. M. Vershik, St. Petersburg Department of Steklov Institute of Mathematics, 27 Fontanka, 191023 St. Petersburg, Russia

E-mail address: vershik@pdmi.ras.ru

M. I. Graev, Institute for System Studies, 36-1 Nakhimovsky Pr., 117218 Moscow, Russia E-mail address: graev_36@mtu-net.ru 